\title{
Native Apps vs. Mobile Web Apps
}

\author{
http://dx.doi.org/10.3991/ijim.v7i4.3226 \\ William Jobe \\ Stockholm University, Stockholm, Sweden
}

\begin{abstract}
The extensive growth and expansion of smartphones and tablets and therewith the use of mobile web applications that utilize HTML5 and related technologies are frequently discussed and debated in media as possible replacements for native applications. The aim of this study was to explore the viability of replacing native applications with mobile web applications in a developing country setting. Two mobile web applications were developed. The first mobile web application tracked runs and the second mobile web application was a booking system for scheduling "slum runs". The subjects who tested these apps were elite, semi-professional Kenyan runners primarily from the Kibera slum area outside of Nairobi. After a 6-month test period the participants concluded and results indicated that the mobile web application for tracking runs performed poorly compared to native applications due to poor GPS performance, while the mobile web application for booking slum runs performed well. The conclusion from this study is that mobile web applications that require hardware interaction such as using the GPS, GPU, or camera are not yet viable alternatives for native applications. However, mobile applications that only require a native interface and content consumption are suitable substitutes for native applications.
\end{abstract}

Index Terms-Mobile web apps, native apps, HTML5, Responsive Web Design, ICT4D

\section{INTRODUCTION}

The immense growth and popularity of smartphones is a global phenomenon and the popularity of such devices continues to expand. However, statistics show that despite the existence of 5 billion mobile subscribers around the world, there are only 1 billion smartphone users, but the market is growing roughly $42 \%$ per year [1]. Moreover, the use of native applications (native apps) on mobile devices such as smartphones and tablets is now universal. In six years, since the advent of the first iPhone, the installation and use of applications has become a given and trivial process when using any modern mobile device. The application repository concept and its usage is basically the same despite that existence of two dominant, yet exclusive mobile operating systems, Apple's iOS and Google's Android, with a slowly gaining third actor in Windows Phone. It is estimated that roughly 56 billion smartphone apps will be downloaded in 2013 with approximately 58\% being for Android, 33\% for iOS, and the remainder for Windows Phone and BlackBerry [2].

The use of mobile web sites and mobile web applications (mobile web apps), on the other hand, is somewhat more elusive to measure and estimate as there are no app stores so to speak. However, a study that measured the number of web page views showed that $10 \%$ originated from mobile devices globally [3]. This statistic indicates a smaller yet significant usage of the Internet from mobile web browsers. Furthermore, the key technology that mobile web apps depend on is HTML5. The advent of HTML5 and interrelated technologies such as CSS3 and JavaScript APIs has made these common web tools more powerful and capable to produce web apps that rival native apps in terms of functionality, design, interaction, and use of multimedia. Though it is difficult to accurately measure current HTML5 usage, a recent survey of developers showed that $82 \%$ of developers found HTML5 important to their jobs within the next 12 months, $63 \%$ were currently using HTML5, and $31 \%$ were planning to start [4].

Mobile devices are also commonplace in developing countries and ubiquitous in Kenya. Kenya has around 29 million mobile subscribers and mobile penetration is around $75 \%$ [5]. Internet access in Kenya is around $27 \%$ and $15 \%$ access the Internet via smartphones and the usage of mobile devices and the Internet is increasing [6]. In addition, it is estimated in one study that $54 \%$ of Kenyans never or infrequently use a desktop computing device to access the web, i.e. they only access the web via a mobile device [3]. Additionally, Nicolaou [7] estimates that by 2017 mobile traffic will exceed 11 exabytes of data per month. These statistics reinforce the notion that mobile device and web usage are already very high in Kenya and that the emergence and growth of smartphones have started.

Finally, web searches for "web apps vs. native apps" quickly elucidates the general problem area and the depth and degree of the discussion as to which technology will succeed and why. There exists a great deal of discussion and speculation, but little concrete research or studies. One climax of this speculation is the now infamous Facebook experiment where HTML5 was ditched for native apps [8]. Within this general area of interest, the specific problem area this research addresses is to ascertain whether or not a web app can replace the functionality of a native app considering the fact that mobile phone usage is high in Kenya, but smartphone usage is not yet widespread.

\section{A. Research problem}

Thus, the specific research problem that this study addresses is to examine if the functionality of a mobile app is equivalent to that of native app, and thus provide empirical evidence as to whether they are realistically viable substitutes or not. It web apps can replace native apps, the use of mobile web apps could have widespread consequences in the future growth of mobile development and smart devices in developing countries. Specifically, this research uses design science research methodology and creates two separate mobile web apps, one that accesses specific hardware and one that does not, in order to test and evaluate whether or not mobile web apps are functionally equivalent to native apps. 


\section{RELATED TECHNOLOGIES AND RESEARCH}

A few different variations of mobile applications exist and these are discussed and defined in the following sections. However, there are a number of general aspects of application development and use that each type of app is believed to be better or less suited to solve. These general qualities are listed and explained in Table I below.

TABLE I.

AN EXPLANATION OF HOW NATIVE AND MOBILE WEB APPS PRESUMABLY ADDRESS KEY CHARACTERISTICS OF APP USAGE AND DEVELOPMENT

\begin{tabular}{|c|c|c|}
\hline & Native Apps & Mobile web apps \\
\hline $\begin{array}{c}\text { Create vs. } \\
\text { consume content }\end{array}$ & $\begin{array}{l}\text { Native apps are } \\
\text { more suitable for } \\
\text { content creation due } \\
\text { to performance and } \\
\text { hardware access. }\end{array}$ & $\begin{array}{l}\text { Mobile web apps are } \\
\text { less suited for } \\
\text { content creation, but } \\
\text { equally suited for } \\
\text { content consumption }\end{array}$ \\
\hline User experience & $\begin{array}{l}\text { Seamless integration } \\
\text { with native } \\
\text { operating system }\end{array}$ & $\begin{array}{l}\text { Limited integration, } \\
\text { requires external } \\
\text { frameworks }\end{array}$ \\
\hline $\begin{array}{l}\text { Update } \\
\text { frequency }\end{array}$ & $\begin{array}{l}\text { Updates are formal } \\
\text { through app stores }\end{array}$ & $\begin{array}{lr}\text { Updates are more } \\
\text { informal } \\
\text { equivalent and } \\
\text { website updates }\end{array}$ \\
\hline Performance & $\begin{array}{l}\text { Maximum } \\
\text { performance and } \\
\text { access to device } \\
\text { hardware }\end{array}$ & $\begin{array}{l}\text { Performance is } \\
\text { dependent on } \\
\text { JavaScript rendering } \\
\text { and mobile web } \\
\text { browsers, limited } \\
\text { access to device } \\
\text { hardware }\end{array}$ \\
\hline Functionality & $\begin{array}{l}\text { All functionality in } \\
\text { the mobile operating } \\
\text { system is available }\end{array}$ & $\begin{array}{c}\text { Most of the } \\
\text { functionality of the } \\
\text { mobile operating } \\
\text { system is available }\end{array}$ \\
\hline Development & $\begin{array}{l}\text { Requires specific } \\
\text { development for } \\
\text { each mobile } \\
\text { operating system }\end{array}$ & $\begin{array}{l}\text { Open web languages } \\
\text { and browsers make } \\
\text { "Write once, run } \\
\text { anywhere" } \\
\text { development } \\
\text { possible }\end{array}$ \\
\hline Profitability & $\begin{array}{l}\text { Framework for } \\
\text { monetizing apps is } \\
\text { available via app } \\
\text { stores }\end{array}$ & $\begin{array}{l}\text { No clear, unified } \\
\text { strategy for } \\
\text { monetization. }\end{array}$ \\
\hline
\end{tabular}

\section{A. Native applications}

Native applications refer to applications that are specifically written and developed for a specific mobile operating system. The three leading mobile operating systems are Google's Android, Apple's iOS, and Windows Phone. In order to create true, native applications, the Java programming language must be used for Android, the Objective C programming language for $\mathrm{iOS}$, and the .NET framework for Windows Phone. Common, key characteristics of native applications are that these applications have unhindered access to device hardware and support all user interface and interactions available in the respective mobile operating environment.

\section{B. Dedicated mobile web applications}

Dedicated mobile web applications refer to web applications that are designed and developed to mimic the native applications of the host operating system as much as possible, but they execute in a web browser on the host platform. Dedicated mobile web applications are developed with a combination of HTML5, JavaScript, and CSS.

\section{1) HTML5, CSS, and JavaScript}

HTML5 is the latest standard and current candidate recommendation from the W3C (http://www.w3.org), which is the official, non-profit organization that develops and maintains web standards. HTML5 is candidate recommendation from the $\mathrm{W} 3 \mathrm{C}$ and the official recommended web language to create web pages [9]. HTML5 is both the official recommendation from the $\mathrm{W} 3 \mathrm{C}$ as well as a more informal term used to group the actual HTML5 standard together with the new JavaScript APIs, and CSS3[10]. Key goals of HTML5 are to create a standard with a feature set that can replace proprietary technologies and bring HTML5 into the world of application development [11]. Therefore, all of these technologies modernize the capabilities of these native web languages, so that they offer all the necessary functionality to deliver contemporary web applications to a variety of devices. A short summary of the new functionality and the different levels of approval are listed in Table II below.

TABLE II.

SUMMARY OF NEW FUNCTIONALITIES FOR HTML5 AND RELATED TECHNOLOGIES

\begin{tabular}{|c|c|c|c|}
\hline $\begin{array}{c}\mathrm{W} 3 \mathrm{C} \\
\text { recommenda } \\
\text { tion }\end{array}$ & $\begin{array}{l}\text { Candidate } \\
\text { recommend } \\
\text { ation }\end{array}$ & $\begin{array}{c}\text { Proposed } \\
\text { Recommend } \\
\text { ation }\end{array}$ & $\begin{array}{c}\text { Working } \\
\text { Draft }\end{array}$ \\
\hline MathML & Web storage & GeoLocation & Microdata \\
\hline SVG & $\begin{array}{l}\text { Web } \\
\text { messaging }\end{array}$ & & $\begin{array}{l}\text { XmlHTTP } \\
\text { Request }\end{array}$ \\
\hline Selectors & Canvas 2D & & File API \\
\hline $\begin{array}{l}\text { Navigation } \\
\text { Timing }\end{array}$ & Web sockets & & Media Capture \\
\hline $\begin{array}{l}\text { Open Web } \\
\text { Fonts }\end{array}$ & $\begin{array}{l}\text { Native } \\
\text { audio/video } \\
\text { tags }\end{array}$ & & $\begin{array}{l}\text { Indexed } \\
\text { Database }\end{array}$ \\
\hline \multirow[t]{3}{*}{$\begin{array}{lr}\text { RDFa } & \text { and } \\
\text { HTML } & + \\
\text { RDFa } & \\
\end{array}$} & $\begin{array}{l}\text { New } \\
\text { HTML5 } \\
\text { markup tags }\end{array}$ & & Contacts API \\
\hline & $\begin{array}{l}\text { Drag and } \\
\text { drop }\end{array}$ & & $\begin{array}{l}\text { Device } \\
\text { Orientation }\end{array}$ \\
\hline & Web workers & & $\begin{array}{l}\text { Animation } \\
\text { Timing }\end{array}$ \\
\hline
\end{tabular}

Dedicated mobile web apps, generic mobile web apps, and hybrid web apps all depend on HTML5 and related technologies along with mobile web browsers for rendering in order to deliver web-based applications on a mobile device.

\section{Generic mobile web applications (mobile websites)}

A generic mobile web application is another term for mobile versions of websites. There are a variety of ways to create and develop mobile versions technically, however the usual premise is that the desktop version of a website checks for mobile devices through the user-agent identifier from the web browser. Once a mobile device is detected, the user-agent is redirected either to a dedicated mobile website created for that specific device or to a website that utilizes responsive web design techniques in order to provide the same content to a variety of devices.

\section{1) Responsive web design}

Responsive web design is the concept of using CSS (Cascading Style Sheets), which is a style sheet language for describing the presentation of web pages, and media queries in order to determine the resolution of the device 
being used and adjust the delivery and presentation of the website content accordingly [12]. What responsive web design basically implies is that the use of device specific apps or web applications becomes unnecessary because the content is simply manipulated according to the CSS3 directives provided in order to adapt the content for the screen size of each device. Furthermore, responsive web design even expands/shrinks the content to use available space when the web browser window is resized.

\section{2) jQuery mobile}

jQuery Mobile is a JavaScript library or mobile framework that enables and supports touch events and design elements for a wide variety of tablets and smartphones in order to make them look and function like native apps. jQuery Mobile is developed and maintained by the jQuery project team and is compatible with all major mobile platforms and desktop browsers. It even offers a theming framework that allows web apps to customize aspects of the user interface and CSS in order to imitate the user interface of the host operating system.

\section{Hybrid apps}

A hybrid web app is an application that is neither truly a mobile web app nor a native app. It is basically an application written with the aforementioned web techniques of HTML5, JavaScript APIs, and CSS, but it runs inside a 3 rd party native app container. The key characteristics of a hybrid app are that they are developed with standard web languages, but typically have access to the native device APIs and hardware. Some of the wellknown and used hybrid mobile frameworks are PhoneGap, Appcelerator, and Appspresso.

\section{E. Related research}

Huy and Thanh [13] developed four different applications; a native app, a HTML5 web app, a widget, and a generic mobile web app, and evaluated them on a variety of criteria to try and determine the optimal paradigm for development. Their conclusions were that native apps were fast and responsive but were complicated to develop and required much effort. Furthermore, jQuery Mobile in combination with HTML5 provided an attractive and adaptive user interface. Finally, Huy and Thanh [13] concluded that native apps and HTML5 mobile apps were still the leading mobile paradigms, though distinctions were made between the two.

Hamou et al. [14] performed a study where iPhone web apps were used to collect patient data. Their results showed such web applications were viable replacements for equivalent functionality from websites and were successful in both consuming and creating content by collecting patient data. Additionally, Sin et al. [15] showed that the development of web apps was simple and could be performed by "non-programmers" and offered a user experience comparable to a native app.

Juntunen et al. [16] studied drivers and constraints of HTML5 and found that web apps did not currently offer the usability and added value of native apps, but the gap between native and web apps was closing. Additionally, they stated that the lower costs and cross-platform traits of web apps might prove crucial in the future.

Finally, Costello and Proshaska [17] pointed out that most of the criticisms regarding HTML5 and web apps stems from game developers not corporate app developers, and that the fate and possible success of HTML5 might depend more on digital rights management and platform specific lockdown than anything else.

\section{RESEARCH DESIGN AND METHODS}

The research methodology used in this study was design science research. Hevner et al. [18] define design science research as designing and testing an IT artifact in order to test and/or solve an unsolved problem. In the case of this study design science methods directly align with the goals of this study, which again were to test and evaluate if the functionality of mobile web apps were equivalent to that of native apps and therefore realistically viable substitutions. Specifically, a mobile web app artifact instantiation, a term defined by Hevner et al. [18], was created and evaluated against preexisting native apps with equivalent functionality in an attempt to answer the question as to whether or not mobile web apps could replace native apps.

As Hevner et al. [18] mention, design science is both a process and a product. The process consists of building and evaluating and the product is the actual IT artifact. In the case of this study, two separate and distinct mobile web apps represented the IT artifacts that were built and evaluated. These mobile web apps were developed using the aforementioned technologies of HTML5 and Mobile jQuery without any other external or hybrid APIs. The first mobile web app tracked runs and thereby was dependent on accessing the GPS hardware in the smartphone to work properly. This web app provided the standard functionality of similar native apps in that you could start, stop, pause, and record runs as well as view your progress and completed run on a map. The second web app was of a more generic nature in that it allowed users to consume content by learning about "slum runs", i.e. guided runs through slum areas that are a variation on more established slum walks, and create content on a limited scale by booking a "slum run" directly from a smartphone by choosing a specific date and time to meet with a running guide.

The evaluation process of the two mobile web apps consisted of testing the mobile web apps as well as equivalent native apps in a realistic setting. The chosen participants were Kenyan runners already partaking in a yearlong (Nov. 2011 - Nov. 2012) research project entitled the FrontRunner project. This project had a target group that consisted of runners from the slum of Kibera in Nairobi (East Africa's largest slum) and from Ngong town (20 km outside Nairobi). This project focused on how a smartphone affected the runners' informal learning, business opportunities, and training. The runners were chosen as the primary target group for evaluating the different applications because they had little formal education, and they were a close-knit social group with no previous experience with smartphones. In total there were 30 runners ( 21 men and 9 women) from 19 to 34 years of age, and the majority had not completed secondary school. All of the selected runners were part of a larger training group, so for the FrontRunner project they were chosen by their coach to participate. The runners were chosen based on their performance and attendance in training. The runners were semi-elite (in terms of racing results just below the elite level), elite on the national level, or worldclass elite (competing professionally in international races). 
All the runners in the target group already had a simple mobile phone, but they had never used a smartphone. All 30 Kenyan runners were equipped with a simple (\$80 cost) Android smartphone (Huawei Ideos) and free Internet time (1.5 GB traffic/month). The research institutions backing this research effort paid for the smartphones and Internet time. 29 of the 30 smartphones were successfully tracked, and all aspects of telephone usage were recorded by a locally installed app and sent to servers when a data connection was available. Due to a variety of technical issues, all activity was not successfully logged for the entire year. However, the tracking for all 29 smartphones was for at least 4-6 months of the entire period, and 3 smartphones were tracked for the entire time period. This tracking meant that the number of text messages, calls, GPS-locations, applications used, and web pages accessed were recorded and stored for each runner. The tracking log data even provided the specific dates and times of use. This concrete data supplied important, objective information that balanced the subjective images that emerged in the formal interviews, as well as provided security measures if the telephone were lost or stolen. The participants were well aware of the tracking, and it was thoroughly discussed both within the groups and with the researchers. Permission from each runner was given in a written, informed consent letter.

Naturally tracking and logging personal information creates an ethical discussion of the research design, and it was continuously discussed during workshops, meetings, and interviews. Our educational institutions approved the informed consent forms in October of 2011. In November of 2011, at the start of the study in combination with dispersal of the smartphones, the runners first read the informed consent letter themselves. They then had the informed consent forms read aloud and thoroughly explained to them in both English and Kiswahili before signing. In addition, the exit strategy for this study was that after the research period expired (Nov. 2012), the participants were allowed to keep the smartphones to use as they wished.

The runners received the aforementioned Huawei Ideos Android smartphones already configured with a direct link to the web run-tracking app on their homes screens. These smartphones used the Android 2.3 Gingerbread operating system, and the runners used the default web browser when testing the mobile web apps. In addition, the two most popular, free run-tracking native apps at the time, RunKeeper and MyTracks, were installed on the runners' smartphones. The runners were then instructed to use and test the basic functionality of the mobile web apps and native apps when training and competing. A key benefit to having the Kenyan runners evaluate the apps, in addition to their expertise as runners, was that they had no previous experience with smartphones, native apps, or mobile web apps of any kind. Finally, they were told to test all three running apps equally and choose the one they deemed best to accurately track and record runs.

The same runners also evaluated the second mobile web app for booking "slum runs". A link was created to the mobile web app for booking "slum runs", and the Kenyan runners as well as those who booked "slum runs" from their smartphones evaluated this app. The evaluation of this app focused on simply determining if the functionality of the app was sufficient to quickly and accurately book "slum runs" from a smartphone. Additionally, the researchers tested all the apps from Europe as well, in order to discover possible differences that might be encountered when the apps used different GPS satellites. The runners were then interviewed in groups regarding their usage of the different apps along with their impressions and experiences of both. Finally, the tracking log data were analyzed to see how often the mobile web apps and native apps were actually used.

\section{RESUlts}

The results are categorized according to the two different tests of mobile web apps. The first section presents the results for the run-tacking mobile web app. The second section presents the results for the mobile web app for booking "slum runs".

\section{A. Mobile web app for tracking runs}

The tracking log data showed that the runners used the run-tracking mobile web app a total of 313 times for all of them, which was roughly 10 times per runner. During subsequent interviews the runners expressed the same general sentiment, which was that they had tested the mobile web app as instructed, but the GPS location data was always incorrect. The results showed that the GPS location functionality jumped a few hundred meters in different directions and therewith added distance to the run without having actually started to run. However, the runners stated that after this initial error, the tracking generally took place correctly and was more or less equivalent to the native run-tracking apps. Tests made in Europe by the researchers using the same mobile web app and hardware reaffirmed these results. An example of the appearance of the run-tracking mobile web app is shown in Figure 1.

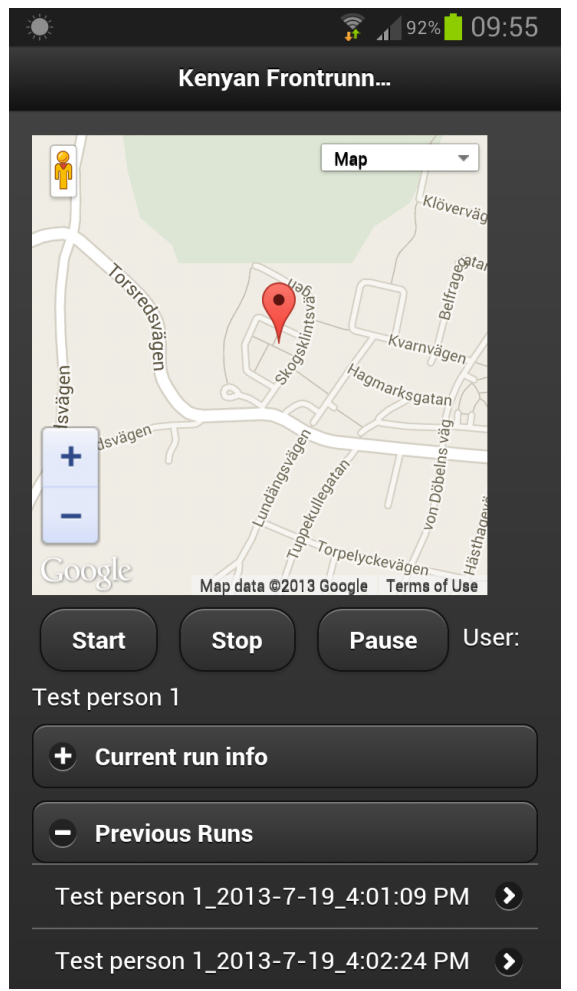

Figure 1. An example of the mobile web app for tracking runs. 
Because the runners obviously took the times and distances of runs very seriously, they eventually quit using the mobile web app due to the constant miscalculation of the distance. Therefore, they switched instead to using the two native run-tracking apps, MyTracks and RunKeeper, which were preinstalled before they received their smartphones. The tracking log data showed that the runners used MyTracks a total of 2,517 times and RunKeeper a total of 2,397 times. These numbers broken down per runner meant that each runner used MyTracks 86 times and RunKeeper 82 times. However, in reality some runners ran more than others obviously. When asked why they chose the native apps instead of the mobile web app, the runners stated that the native apps were more accurate and faster. During subsequent interviews with the runners, they explained that it was necessary to use the native apps because they tracked the distance better. The runners also stated that the mobile web app was usually slower and more sluggish than the native apps and took longer to locate the GPS signal.

\section{B. Mobile web app for booking "slum runs"}

The mobile web app for booking a "slum run" provided information about "slum runs" and the runners, and allowed users to book "slum runs". Booking a "slum run" took place by providing a name and email address as well as suggesting a date and time for the actual run. When a preliminary booking was made, an email was sent to the runners' email accounts in their smartphones.

According to the tracking log data, the mobile web app for booking "slum runs" was accessed over 300 times by the runners. However, it was only used to book five "slum runs" during the research time period. Access by users, who were not runners in this research group, was not recorded. During interviews the runners stated that many more "slum runs" were booked face-to-face, and the runners instructed prospective runners to www.slumrun.com to use the mobile web app. Since the mobile web app also provided general and specific information about "slum runs", the Kibera slum, and the runners themselves, the mobile web app was used more often for information gathering than simply for booking "slum runs". An example of the mobile web app for booking "slum runs" is shown in Figure 2.

During interviews, all the runners agreed that the mobile web app for booking "slum runs" functioned adequately, and they all felt that the ability to provide an URL to the booking system made it very easy to advertise for bookings and make information available about "slum runs". Also, the runners stated that the fact that the mobile web app worked on a variety of devices, even older feature phones and desktops, made the mobile web app even more useful. Lastly, the runners had no direct complaints or issues with the functionality or performance of the mobile web app for booking "slum runs".

\section{DISCUSSION}

The results were clear and unanimous regarding the mobile run-tracking web app versus the native runtracking apps. The runners observed that the GPS location was too inaccurate at the beginning of the runs. This result confirmed the speculation regarding the performance and functionality of mobile web apps versus native apps, which was that mobile apps could not perform as well as native apps due to the lack of direct access to the device

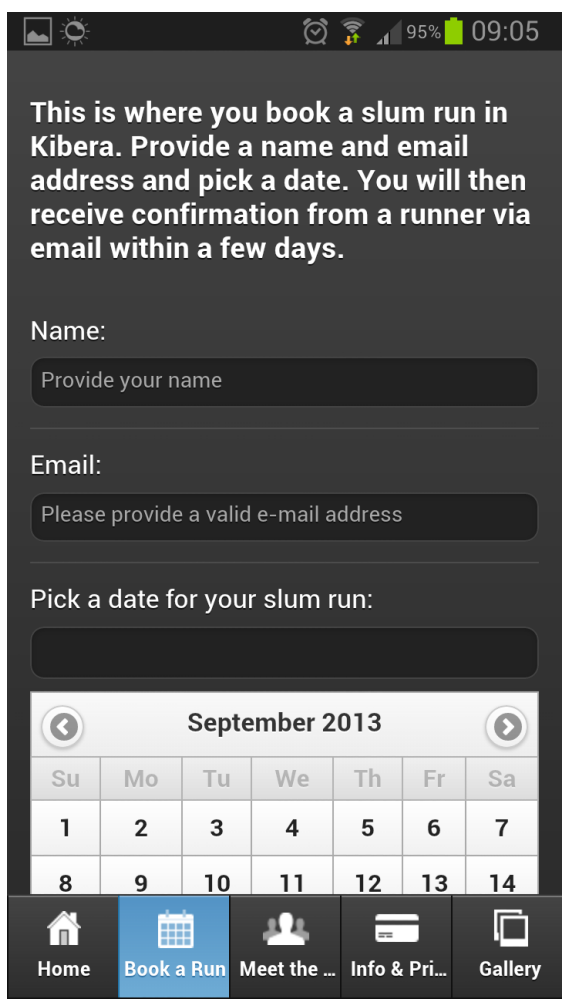

Figure 2. An example of the mobile web app for booking "slum runs"

hardware. This conjecture proved true in this case and hints that other device intensive applications would be better served by using native apps. This result reaffirmed Huy and Thanh [13] results where they stated that hardware intensive applications such as gaming applications were best suited for native apps.

Despite the fact that the mobile run-tracking web app performed more poorly than the native app, the complexity and time needed to develop the web app was minimal compared to the investment in time, money, and man-hours for native apps such as MyTracks and RunKeeper. The choice of app development does not solely depend on performance and functionality. Sometimes "good enough" performance and functionality could be sufficient to satisfy the needs of the user without making a significant investment. Furthermore, the ability to develop adequate web apps quickly and inexpensively could be extra beneficial in developing countries. One study by the Kenyan ICT board [6] found that there is a limited supply of skilled ICT workers and the ability to develop applications with native web languages for all devices instead of proprietary languages for specific devices could alleviate the need for skilled ICT labor. Finally, the use of hybrid apps as a viable alternative should be studied as well. Hybrid apps may present the best path to achieve higher performance and functionality with reduced investment costs in both developed and developing countries.

However, the mobile web app for booking "slum runs" was appreciated by the runners and proved to be highly useful. This web app showed that applications that primarily focused on content consumption could be seamlessly replaced by web apps. The runners used the web app as if it were a native app without any significant problems. The only noteworthy drawback mentioned was sluggishness, but this issue was related to the rendering of 
JavaScript in the web browser and was a known issue [7]. In the future this issue will be minimized automatically due to the constant improvements to JavaScript rendering in web browsers. Another possible issue regarding this web app was that the customers who used the web app for actually booking "slum runs" were not interviewed and the researchers only performed precursory discussions with a few potential customers on site in Kenya. This group of users could be studied in greater detail in order to draw better conclusions regarding performance and functionality.

Another possible drawback to the viability of using a mobile web app instead of a native app in the setting of a developing country was access to the Internet. Access to the Internet in Kenya and many other developing countries was often adequate but too costly, especially for the poor. The mobile web apps for tracking runs and booking "slum runs" did not cache maps and other content for offline use. Consequently, the native apps used less data and therefore cost less. Mobile web apps in developing countries need to prioritize caching and offline mode in order to minimize Internet data usage and costs.

These results reaffirmed the findings of Huy and Thanh [13] regarding paradigms for mobile development, but also extended the categorization and utility of mobile web apps into mobile web apps that were hardware intensive and primarily for creating content, and mobile web apps that did not require hardware access and were primarily for content consumption.

\section{CONCLUSIONS}

The conclusions from this research are that native apps are still the best choice for hardware intensive apps. However, websites or applications that primarily consume content can be successfully replaced with web apps. Therefore, web apps are viable substitutes for native apps in such use cases. In a context of a developing country such as Kenya, this means that using web apps for content consumption can drastically reduce issues such as high development costs and problems finding professional developers. Furthermore, with the ongoing development of a W3C device API to directly access hardware the limitation on using web apps for hardware intensive applications should be reduced in the future.

This research provides early results on a small scale that are not directly generalizable for larger populations. Therefore, there is a need for broader and lengthier studies to explore the extent to which mobile apps that primarily consume content can replace native apps. This research could even focus on various aspects of user interaction in the two application paradigms. Future research should focus on further exploring how other device intensive mobile web apps perform versus native apps. Finally, though there is a need for future research to test the feasibility of HTML5 and web apps for app usage in developing countries, this research has provided an indication of the possibilities of an impending breakthrough for developing mobile web apps.

\section{REFERENCES}

[1] P. Olson, "5 Eye-Opening Stats That Show The World Is Going Mobile," Forbes, 04-Dec-2012. [Online]. Available: http://www.forbes.com/sites/parmyolson/2012/12/04/5-eye- opening-stats-that-show-the-world-is-going-mobile/. [Accessed: 05-Sep-2013].

[2] dotMobi, "Global mobile statistics 2013 Section E: Mobile apps, app stores, pricing and failure rates," mobiThinking, May-2013. [Online]. Available: http://mobithinking.com/mobile-marketingtools/latest-mobile-stats/e\#appdownloads. [Accessed: 06-Sep2013].

[3] dotMobi, "Global mobile statistics 2013 Part B: Mobile Web; mobile broadband penetration; $3 \mathrm{G} / 4 \mathrm{G}$ subscribers and networks.," mobiThinking, May-2013. [Online]. Available: http://mobithinking.com/mobile-marketing-tools/latest-mobilestats/b\#mobilepageviews. [Accessed: 06-Sep-2013].

[4] Kendo UI, "HTML5 Adoption Fact or Fiction," Sep. 2012.

[5] Communications Commission of Kenya, "Quarterly Sector Statistics Report," Communications Commission of Kenya, Aug. 2012.

[6] Kenya ICT Board, "Julisha Survey on Kenya ICT Market Overview 2011 | Open Kenya | Transparent Africa," Open Kenya, 2011. [Online]. Available: https://opendata.go.ke/Education/ Julisha-Survey-on-Kenya-ICT-Market-Overview-2011/3j44-wqzt. [Accessed: 13-May-2013].

[7] A. NICOLAOU, "Best Practices on the Move: Building Web Apps for Mobile Devices.," Commun. ACM, vol. 56, no. 8, pp. 45-51, Aug. 2013. http://dx.doi.org/10.1145/2492007.2492023

[8] J. J. Colao, "Facebook's HTML5 Dilemma, Explained," Forbes, 19-Sep-2012. [Online]. Available: http://www.forbes.com /sites/jjcolao/2012/09/19/facebooks-html5-dilemma-explained/. [Accessed: 09-Sep-2013].

[9] W3C, "HTML5," W3C Candidate Recommendation, 06-Aug2013. [Online]. Available: http://www.w3.org/TR/htm15/. [Accessed: 05-Sep-2013].

[10] R. Larsen, "HTML5, CSS3, and related technologies," developerWorks, 26-Apr-2011. [Online]. Available: http://www.ibm.com/developerworks/library/wa-webstandards/. [Accessed: 26-Sep-2013].

[11] S. J. Vaughan-Nichols, "Will HTML 5 Restandardize the Web?," Computer, vol. 43, no. 4, pp. 13-15, 2010. http://dx.doi.org/10.1109/MC.2010.119

[12] E. Marcotte, "Responsive Web Design," A List a Part, 2010. [Online]. Available: http://alistapart.com/article/responsive-webdesign. [Accessed: 15-May-2013].

[13] N. P. Huy and D. van Thanh, "Selecting the right mobile app paradigms," in 2012 5th IEEE International Conference on Service-Oriented Computing and Applications (SOCA), 2012, pp. $1-6$.

[14] A. Hamou, S. Guy, B. Lewden, A. Bilyea, F. Gwadry-Sridhar, and M. Bauer, "Data collection with iPhone Web apps efficiently collecting patient data using mobile devices," in 2010 12th IEEE International Conference on e-Health Networking Applications and Services (Healthcom), 2010, pp. 235-239.

[15] D. Sin, E. Lawson, and K. Kannoorpatti, "Mobile Web Apps - The Non-programmer's Alternative to Native Applications," in 2012 5th International Conference on Human System Interactions (HSI), 2012, pp. 8-15.

[16] A. Juntunen, E. Jalonen, and S. Luukkainen, "HTML 5 in Mobile Devices - Drivers and Restraints," in 2013 46th Hawail International Conference on System Sciences (HICSS), 2013, pp. 1053-1062.

[17] T. Costello and B. Prohaska, "2013 Trends and Strategies," IT Prof., vol. 15, no. 1, pp. 64-64, 2013. http://dx.doi.org/ 10.1109/MITP.2013.5

[18] A. R. Hevner, S. T. March, J. Park, and S. Ram, "Design science in information systems research," $M I S Q$, vol. 28 , no. 1, pp. $75-$ 105, Mar. 2004

\section{AUTHOR}

William Jobe is with Stockholm University, Stockholm, Sweden.

Submitted 12 September. Published as re-submitted by the author 12 October 2013. 